\title{
Zur Methodik der Ammoniakbestimmung. Von
}

\author{
Alfred Schittenhelm.
}

(Aus der medizinischen Klinik der Universität Breslau.) (Der Redaktion zugegangen am 9. Juni 1903.)

Mit der alten Schlösing-Neubauerschen Meth́ode der Ammoniakbestimmung werden nur annähernde Werte gefunden. Dieselben sind zwar für den Ammoniakgehalt des Urins noch relativ brauchbare, zu der Bestimmung des Ammoniakgehaltes von Blut. und Geweben ist die Methode jedoch so gut wie nicht verwendbar. Es haben sich daher in den letzten Jahren verschiedene Forscher mit der Methodik der Ammoniakbestimmung beschäftigt, in der Absicht, an Stelle der mehrere Tage dauernden und ungenauen alten Methode eine genügend genaue und schnell auszuführende neue zu setzen.

W urster ${ }^{1}$ ) suchte diese Übelstände durch die Destillation im Vacuum zu beseitigen, eine Methode, wie sie mehrere Jahrzehnte früher schon von Boussingnault ${ }^{2}$ ) angegeben war, ohne daß sie jedoch damals eine verbreitetere Anwendung gefunden hätte. Söldner ${ }^{3}$ ) und Nencki und Zaleski ${ }^{4}$ ) haben die Methode modifiziert. Der relativ komplizierte Apparat, welcher auch in seinen Modifikationen nicht vereinfacht ist, war wohl der Hauptgrund, weshalb die Methode keine allgemeine Anwendung gefunden hat. Eine von $\mathrm{Folin}^{5}$ ) angegebene

1) Zentralblatt f. Physiologie 1887, S. 485.

2) Journal f. prakt. Chem., Bd. 51, S. 281 (1850).

3) Zeitschr. f. Biologie, Bd. 38, S. 237.

4) Arch. f. experim. Pathol. u. Pharmak., Bd, XXXVI, S. 385.

s) Diese Zeitschr., Bd. XXXII und XXXVII. 
Methode ist inzwischen von dem Autor selbst als fehlerhft erkannt worden.

Krüger und Reich ${ }^{1}$ ) haben eine Modifikation dr Wursterschen Methode angegeben, welche, da sie bis jett nur in der Dissertation von Reich publiziert ist, offenbar noh nicht weiter bekannt geworden ist. Die Modifikation bestet in der Verwendung von Alkohol als Zusatz bei der Vacuundestillation, wodurch einerseits die Siedetemperatur heragesetzt, andererseits das lästige Schäumen des mit Alkali vesetzten Urins so sehr vermindert wurde, daß eine Vereilfachung des Apparates möglich wurde. Die Methode ist de folgende: In einem Destillationskolben werden $25 \mathrm{ccm}$ Han mit $10 \mathrm{ccm}$ Kalkmilch und $10 \mathrm{ccm}$ säurefreiem 96\% \% igen Akohol gemischt und nun mit der als Vorlage dienenden Peigotschen Röhre verbunden, welche $25 \mathrm{~cm} \mathrm{n/10} \mathrm{Normalsalzsäue}$ enthält und gut in Eis gekühlt ist. Der zweite Schenkel der Peigotschen Röhre ist mit der Wasserstrahlpumpe verbundel. Die Destillation geschieht durch Erhitzen des ersten Kolbeis im Wasserbade, dessen Temperatur $43^{\circ}$ nicht überschreiten sol, unter einem Druck von 30-40 mm Quecksilber. 17 Minuten nach Beginn des lebhaften Siedens ist die Destillation beendet. Dann läßt man zum Schlusse durch einen am Destillation;kolben angebrachten Quetschhahn $10 \mathrm{ccm}$ Alkohol zu, welchır den Kolbeninhalt wieder in lebhaftes Sieden bringt und die $n$ der Überleitungsröhre befindlichen Wassertropfen wegspült. Jetat wird die Wasserstrahlpumpe nach vorherigem Abklemmen ihrær Verbindung mit der Peligotschen Röhre abgestellt, darauf durch den am Destillationskolben angebrachten Quetschhahn die Luft in den Kolben und in die Vorlage eingelassen und endlich der Inhalt der Peligotschen Röhre mit $\mathrm{n} /{ }_{10}$ Normalnatronlauge zurücktitriert, wobei Rosolsäure als Indikator benutzt wird.

Vor kurzem hat Folin $^{2}$ ) eine neue Methode zur Bestimmung des Ammoniaks im Harne und anderen tierischen Flüssigkeiten angegeben. Dieselbe beruht darauf, daß aus der

1) J. A., Breslau 1902.

2) Diese Zeitschr., Bd. XXXVII, S. 161. 
das Ammoniak enthaltenden Flüssigkeit, nach Zusatz eines schwachen Alkalis, wie Natriumcarbonat oder Calciumhydrat, das freigesetzte Ammoniak bei Zimmertemperatur oder sogar in der Kälte durch einen starken Luftstrom ausgetrieben wird. Der Luftstrom muß ziemlich stark sein (600-700 l per Stunde), um aus 25 oder $50 \mathrm{ccm}$ Flüssigkeit alles Ammoniak in $1-11^{1 / 2}$ Stunden auszutreiben. Ich will hier sofort bemerken, daß ich mit dieser Methode keine Kontrollversuche anstellen konnte, da sich bald ergab, daß unsere Wasserstrahlluftpumpen offenbar keinen genügend starken Luftstrom erzeugen konnten, um alles Ammoniak auszutreiben.

Da ich mich zum Zwecke ausgedehnter Untersuchungen des Ammoniakgehaltes vom Urin ${ }^{1}$ ) der Krüger-Reichschen Modifikation der Wursterschen Methode bedienen wollte, habe ich dieselbe einer Kontrolle unterzogen, wobei sich ergab, daß sie für den Urin ausgezeichnete Resultate liefert. Eine Reihe von 20 Doppelbestimmungen in je $30 \mathrm{ccm}$ Harn ergab als höchste Differenz zwischen den einzelnen Versuchen $0,2 \mathrm{ccm}$ ${ }^{1} /{ }_{10}$ Normalsäure, entsprechend $0,34 \mathrm{mg}$ Ammoniak, als mittlere Differenz aber nur $0,081 \mathrm{ccm}{ }^{1} / 10$ Normalsäure $=0,1377 \mathrm{mg}$ Ammoniak. Höhere Differenzen als $0,2 \mathrm{ccm} 1 / 10$ Normalsäure wurden nie erhalten. Beim nochmaligen Destillieren des Rückstandes von drei verschiedenen Versuchen durch 20 Minuten hindurch nach Zusatz von weiteren $10 \mathrm{ccm}$ Kalkmilch, $15 \mathrm{ccm}$ Wasser und $20 \mathrm{ccm}$ Alkohol wurden für Ammoniak verbraucht: $0,07,0,05,0,05 \mathrm{~cm} \mathrm{1/10} \mathrm{Normalsäure.} \mathrm{Es} \mathrm{genügte} \mathrm{also} \mathrm{ein}$ Tropfen der $1 / 10$ Normalsäure zur Bindung des bei der Nachdestillation übergegangenen Ammoniaks. Hiernach kann eine Zersetzung stickstoffhaltiger Körper im Urin durch diese Methode ausgeschlossen werden und ich habe daher von Kontrollversuchen mittels Harnstofflösungen u.s.w. um so eher Abstand genommen, als Reich schon genaue Versuche damit anstellte. Meine Versuche stimmen mit den von Reich ausgeführten gut überein. Es ist demnach die Krüger-Reichsche Methode

1) Die Arbeit wird in einem der nächsten Hefte des Archivs für klinische Medizin erscheinen. 
eine für die Ammoniakbestimmung im Urin durchaus vollkommene, welche vor allem infolge ihrer einfachen Ausführung sich für klinische $Z$ wecke vorzüglich eignet.

Die Anwendung der Methode zur Bestimmung des Ammoniakgehaltes der Faeces stieß dagegen auf Schwierigkeiten, insofern sich ergab, daß dabei eine gleichmäßig verlaufende Zersetzung stickstoffhaltiger Körper stattfand. $\mathrm{Zu}$ diesen Versuchen zerrieb ich ganz frische Faeces in der Reibschale aufs feinste mit 1/2\% iger Salzsäurelösung. Darauf füllte ich das Ganze auf ein bestimmtes Volumen auf und nahm davon zu den einzelnen Bestimmungen $30-50 \mathrm{ccm}$. Die Doppelbestimmungen mittels der KrügerReichschen Methode ergaben im Anfang scheinbar übereinstimmende Resultate. Als ich jedoch den Rückstand mehrmaliger Nachdestillation unter nochmaligem Zusatz von Wasser, Kalkmilch und Alkohol unterwarf, ergab sich, daß von $30 \mathrm{zu}$ 30 Minuten stets genau eine Ammoniakmenge frei wurde, welche $1 \mathrm{ccm} \mathrm{1/10} \mathrm{Normalsäure} \mathrm{entsprach.} \mathrm{Ein} \mathrm{Versuch,} \mathrm{diesen}$ Mißstand durch eine Herabsetzung der Temperatur zu heben, indem ich an Stelle des Äthylalkohols Methylalkohol verwandte und anfangs bei $36^{\circ}$, dann bei $33^{\circ}$ destillierte, mißlang, indem sich sofort zeigte, daß auch hier eine gleichmäßige Zersetzung von stickstoffhaltigen Körpern, wenn auch in weniger breitem Maßstabe, stattfand. Ich erhielt bei den Nachdestillationen Ammoniakmengen, welche 0,37, 0,41 und 0,31 usw. ccm $1 / 10$ Normalsäure entsprachen. Es ist daraus ersichtlich, daB nicht die Temperatur, sondern wahrscheinlich das angewandte Alkali die Schuld am Mißlingen traf.

Beim Suchen nach einem geeigneten Alkali verwandte ich zuerst Baryum-, dann Strontiumhydroxyd, ohne jedoch bessere Resultate zu erzielen. Magnesiumoxyd hatte ebenfalls zersetzende Wirkung. Kupferhydroxyd erwies sich als zu schwach, indem bei dessem Zusatz offenbar nicht alles Ammoniak ausgetrieben wurde.

Ich kam demnach zu demselben Resulsate, zu dem auch Folin gekommen ist, daß nämlich an der Zersetzung stickstoffhaltiger Substanzen die angewandten Alkalien, 
resp. deren Hydroxylionen die Schuld tragen. Folin verwendet deshalb bei seiner Methode Natriumchlorid und krystallisiertes Natriumcarbonat zur Alkalisierung und findet dieselben für sehr geeignet. Meine Beobachtungen stimmen damit gut überein, indem sich des weiteren bei Verwendung von Natriumcarbonat als Alkali die Vacuumdestillation für die verschiedensten Objekte (Urin, Faeces, Blut usw.) als durchweg zuverlässig erwies.

Im Anfang war das Schäumen äußerst lästig. Es zeigte sich jedoch, daß dasselbe durch öfteren Zusatz von $15-20 \mathrm{ccm}$ Alkohol und eventuell, falls die Flüssigkeit zu sehr eingedampft war, von 10-15 ccm destilliertem Wasser leicht vermieden werden konnte. Man muß sich aber beim Zusetzen des Alkohols usw. peinlichst hüten, durch gleichzeitiges Einlassen von Luft Schwankungen des Vacuums herbeizuführen, weil diese sehr intensives Schäumen verursachen. Zur Vermeidung dieser Störung ist vor allem das Glasrohr, durch welches der Alkohol usw. in den Kolben gebracht wird, am unteren Ende in eine Kapillare auszuziehen. Sollte jedoch trotz aller Vorsicht Luft eindringen, so läßt man so lange Alkohol ganz langsam zufließen, bis das Vacuum wieder sein Maximum erreicht hat.

Die Methode gestaltet sich demnach folgendermaßen:

25-50 ccm des auf seinen Ammoniakgehalt zu untersuchenden Objektes, welches eventuell bei fester Konsistenz vorher mit 1/2\% verrieben und auf ein bestimmtes Volumen aufgefüllt wurde, werden im Destillationskolben mit ca $10 \mathrm{~g}$ Natriumchlorid versetzt und darauf soviel Natriumcarbonat zugesetzt, bis deutliche alkalische Reaktion vorhanden ist. Hiezu genügt meist $1 \mathrm{~g}$. Hierauf wird der Kolben ins Wasserbad gesetzt und mit der als Vorlage dienenden, in Eiswasser ruhenden Peligotschen Röhre, in welche vorher $10-30 \mathrm{ccm} 1 / 10$ Normalsäure mit einigen Tropfen Rosolsäure gefüllt worden war, verbunden. An den zweiten Schenkel der Peligotschen Röhre wird nun die Wasserpumpe angeschlossen und sofort so gut wie möglich evacuiert. Sobald das Vacuum den höchsten Grad erreicht hat; 
werden durch den am Kolben angebrachten Quetschhahn ca. $20 \mathrm{ccm}$ Alkohol zugegeben und nun das Wasserbad auf eine Temperatur von ca $43^{\circ}$ gebracht. In der Folge gebe ich von $10 \mathrm{zu} 10$ Minuten 15-20 ccm Alkohol auf dieselbe Weise zu, eventuell auch noch $10-15 \mathrm{ccm}$ Wasser, falls die Flüssigkeit zu rasch eindampft. Zum Schlusse werden zur Verjagung der Wassertropfen in der Überleitungsröhre nochmals $10 \mathrm{ccm} \mathrm{Al-}$ kohol zugegeben. Nach 30-40 Minuten ist die Bestimmung zu Ende geführt. Es wird nun durch einen Quetschhahn die Wasserstrahlpumpe von der Peligotschen Röhre abgeschlossen und darauf durch vorsichtiges Öffnen des an dem Kolben angebrachten Quetschhahns die Luft langsam zum Einströmen gebracht. Trotzdem ein Erhitzen auf $53^{\circ}$ nichts an der Genauigkeit der Methode schadet (s. unten II, c), so empfiehlt es sich doch, das Wasserbad auf einer Temperatur von $43-44^{\circ} \mathrm{zu}$ halten, da mit dem Anstieg der Temperatur das Schäumen an Intensität zunimmt. Ein Absinken der Temperatur unter die angegebene Höhe bedingt natürlich eine verlängerte Zeitdauer der Bestimmung. (Die Abbildung des Apparates findet sich in der Reichschen Dissertation.)

Als Belege für die Methode mögen die folgenden Beispiele dienen:

I. Urin: $\mathrm{Zu} 30 \mathrm{ccm}$ desselben $+10 \mathrm{~g} \mathrm{NaCl}+1 \mathrm{~g} \mathrm{Na}_{2} \mathrm{CO}_{3}$ $+20 \mathrm{C}_{2} \mathrm{H}_{5} \mathrm{OH}$ nach 25 Minuten bei $43^{\circ}$ verbraucht

bei einer Bestimmung $\mathbf{7 , 0 3} \mathbf{1} / \mathbf{1 0}$ Normalsäure (=11,9 $\mathrm{mg} \mathrm{NH}_{3}$ )

" zweiten \$ 6,91 $\quad(=11,7$ " ).

Die zweite Bestimmung nochmals angesetzt mit $10 \mathrm{H}_{2} \mathrm{O}+20$ $\mathrm{C}_{2} \mathrm{H}_{5} \mathrm{OH}$ nach 20 Minuten bei $43^{0}$ verbraucht $\mathbf{0 , 0}{ }^{1} /{ }_{10}$ Normalsäure.

Derselbe Urin nach Krüger-Reichscher Methode: $30 \mathrm{ccm}$ desselben +10 Kalkmilch $+20 \mathrm{ccm} \mathrm{C}_{2} \mathrm{H}_{5} \mathrm{OH}$ nach 25 Minuten bei $43^{\circ}$ verbraucht $6,89^{1} /{ }_{10}$ Normalsäure $\left(=11,7 \mathrm{mg} \mathrm{NH}_{3}\right)$.

II. Faeces: Nach dem Zerreiben mit $1 / \mathbf{2} \%$ oiger HCl-Lösung auf $400 \mathrm{ccm}$ aufgefüllt.

a) $30 \mathrm{ccm}+10 \mathrm{~g} \mathrm{NaCl}+1 \mathrm{~g} \mathrm{Na}_{2} \mathrm{CO}_{3}+20 \mathrm{C}_{2} \mathrm{H}_{5} \mathrm{OH}$ nach 40 Minuten bei $43^{0}$ verbraucht $\mathbf{6 , 1 5}{ }^{1 / 10}$ Normalsäure (= 10,45 mg $\left.\mathrm{NH}_{3}\right)$. 
b) $30 \mathrm{ccm}$ auf dieselbe Art bei $43^{\circ}$ nach 40 Minuten verbraucht 6,25 $\mathrm{ccm}^{1} / 10$ Normalsäure $(=10,6 \mathrm{mg} \mathrm{NH})_{3}$.

c) $30 \mathrm{ccm}$ auf dieselbe Art bei $\mathbf{5 3}^{0}$ nach 40 Minuten verbraucht 6,2 $\mathrm{cm}{ }^{1 / 10}$ Normalsäure $\left(=10,64 \mathrm{mg} \mathrm{NH}_{3}\right)$. Die Nachdestillationen der 3 Versuche ergaben nach nochmaligem Zusatze von $15 \mathrm{H}_{2} \mathrm{O}$ und $20 \mathrm{C}_{2} \mathrm{H}_{5} \mathrm{OH}$ nach je 20 Minuten bei $43^{\circ}$ eine Ammoniakmenge, welche der Reihe nach 0,08, 0,0, $0,02 \mathrm{ccm} 1 / 10$ Normalsäure entsprach. Es war somit alles Ammoniak übergegangen; eine Zersetzung von stickstoffhaltigen Substanzen trat nicht ein.

III. Faeces: Nach dem Zerreiben in der Reibschale auf 800 aufgefüllt.

a) $30 \mathrm{ccm}+10 \mathrm{~g} \mathrm{NaCl}+1 \mathrm{~g} \mathrm{Na} \mathrm{CO}_{3}+20 \mathrm{C}_{2} \mathrm{H}_{5} \mathrm{OH}$ bei $43^{\circ}$ nach 40 Minuten verbraucht. $\mathbf{2 , 3 8} \mathrm{ccm} 1 / 10$ Normalsäure $\left(=4,046 \mathrm{~g} \cdot \mathrm{NH}_{3}\right)$.

b) $30 \mathrm{ccm}+5 \mathrm{ccm}$ einer 1\% 1 igen Chlorammoniumlösung $+10 \mathrm{~g} \mathrm{NaCl}+1 \mathrm{~g} \mathrm{Na}_{2} \mathrm{CO}_{3}+25 \mathrm{ccm}_{2} \mathrm{H}_{5} \mathrm{OH}$ bei $43^{\circ}$ nach 40 Minuten

$$
\begin{aligned}
& \text { verbraucht: } 12,275 \mathrm{ccm} \mathrm{1/10} \mathrm{Normalsäure} \\
& \text { verlangt: } 12,35 \text { » }
\end{aligned}
$$

Die Nachdestillationen der beiden Bestimmungen nach Zusatz von $10 \mathrm{ccm} \mathrm{H}_{2} \mathrm{O}+20 \mathrm{ccm} \mathrm{C}_{2} \mathrm{H}_{5} \mathrm{OH}$ ergaben nach 20 Minuten bei $43^{\circ}$ eine Ammoniakmenge, welche 0,1 resp. $0,12 \mathrm{ccm} 1 / 10$ Normalsäure entsprach. Also auch hier keine Zersetzung.

IV. Ascitesflüssigkeit mit 6\% Albumen:

a) $30 \mathrm{ccm}+10 \mathrm{~g} \mathrm{NaCl}+1 \mathrm{~g} \mathrm{Na}_{2} \mathrm{CO}_{3}+20 \mathrm{C}_{2} \mathrm{H}_{5} \mathrm{OH}$ nach 25 Minuten bei $43^{\circ}$

verbraucht: $0,21 \mathrm{ccm} \mathrm{1/10} \mathrm{Normalsäure.}$

b) $30 \mathrm{ccm}+10 \mathrm{~g} \mathrm{NaCl}+1 \mathrm{~g} \mathrm{Na}_{2} \mathrm{CO}_{3}+20 \mathrm{ccm} \mathrm{C}_{2} \mathrm{H}_{5} \mathrm{OH}$ $+5 \mathrm{ccm}$ einer $1 \%$ oigen Chlorammoniumlösung $+10 \mathrm{~cm} \mathrm{H}_{2} \mathrm{O}$ bei $48^{\circ}$ nach 25 Minuten

$$
\begin{array}{ll}
\text { verbraucht: } & \mathbf{1 0 , 1 0 5} \mathrm{ccm} \\
\text { verlangt: } & \mathbf{1 0 , 1 4}
\end{array}
$$

Die Nachdestillationen nach Zusatz von $10 \mathrm{ccm} \mathrm{H}_{2} \mathrm{O}+$ $20 \mathrm{ccm} \mathrm{C}_{2} \mathrm{H}_{6} \mathrm{OH}$ ergaben nach weiteren 25 resp. 15 Minuten 
80 Alfred Schittenhelm, Zur Methodik der Ammoniakbestimmung.

beidemal einen Verbrauch von $0,01 / 10$ Normalsäure. Also vollkommene Destillation ohne jede Spur von Zersetzung!

V. Hundeblut aus der vena jugularis frisch mit der Spritze entnommen und sofort bestimmt.

a) $1.7 \mathrm{ccm}+10 \mathrm{~g} \mathrm{NaCl}+1 \mathrm{~g} \mathrm{Na}_{2} \mathrm{CO}_{3}+20 \mathrm{~cm} \mathrm{C} \mathrm{C}_{2} \mathrm{H}_{5} \mathrm{OH}$ bei $43^{\circ}$ nach 25 Minuten

verbraucht $0,52 \mathrm{ccm}^{1 / 1}$ o Normalsäure

b) $7 \mathrm{ccm}+5 \mathrm{ccm}$ einer 1\% $1 \%$ igen Chlorammoniumlösung $+20 \mathrm{ccm} \mathrm{H}_{2} \mathrm{O}+20 \mathrm{ccm} \mathrm{C}_{2} \mathrm{H}_{5} \mathrm{OH}+10 \mathrm{~g} \mathrm{NaCl}+1 \mathrm{~g} \mathrm{Na}_{2} \mathrm{CO}_{3}$ nach 25 Minuten bei $43^{\circ}$

verbraucht: $9,99 \mathrm{ccm} 1 / 10$ Normalsäure verlangt: $\mathbf{1 0 , 1 0 9}$,

Die Nachdestillationen der beiden Bestimmungen nach Zusatz von $15 \mathrm{ccm} \mathrm{H}_{2} \mathrm{O}+20 \mathrm{ccm} \mathrm{C}_{2} \mathrm{H}_{5} \mathrm{OH}$ ergaben nach 20 Minuten bei $43^{\circ} 0,03$ resp. 0,0 ${ }^{1} / 10$ Normalsäure als verbraucht. Also auch hier vollkommene Destillation ohne Zersetzung!

Die erhaltenen Ammoniakwerte des Blutes stimmen mit den von Folin angegebenen gut überein. 